\title{
Nasal Cavity Adenocarcinoma
}

National Cancer Institute

\section{Source}

National Cancer Institute. Nasal Cavity Adenocarcinoma. NCI Thesaurus. Code C6015.

An adenocarcinoma that arises from the nasal cavity. 